\title{
XRCC3 Thr241Met polymorphism and ovarian cancer risk: a meta-analysis
}

\author{
Yulan Yan • Hongjie Liang • Ruolin Li • Li Xie \\ Meng Li $\cdot$ Shan Li $\cdot$ Xue Qin
}

Received: 23 September 2013 / Accepted: 23 October 2013 / Published online: 20 November 2013

(C) The Author(s) 2013. This article is published with open access at Springerlink.com

\begin{abstract}
Genetic polymorphism of X-ray repair crosscomplementing group 3 (XRCC3) Thr241Met has been implicated to alter the risk of ovarian cancer, but the results are controversial. In order to get a more precise result, a metaanalysis was performed. All eligible studies were identified through an extensive search in PubMed, Excerpta Medica Database (Embase), Chinese National Knowledge Infrastructure database, and Chinese Biomedical Literature Database before August 2013. The association between the XRCC3 Thr241Met polymorphism and ovarian cancer risk was conducted by odds ratios (ORs) and $95 \%$ confidence intervals (95\% CIs). Finally, a total of four publications including seven studies with 3,635 cases and 5,473 controls were included in our meta-analysis. Overall, there was no association between XRCC3 Thr241Met polymorphism and risk of ovarian cancer under all five genetic models in overall population (T vs. $\mathrm{C}: \mathrm{OR}=0.99,95 \% \mathrm{CI}=0.960-1.03, P=0.752$; $\mathrm{TT}$ vs.
\end{abstract}

Yulan Yan and Hongjie Liang contributed equally to this work, so they should be considered as the co-first authors.

Y. Yan $\cdot$ H. Liang $\cdot$ R. Li $\cdot$ L. Xie $\cdot$ M. Li $\cdot$ S. Li $(\bowtie) \cdot$ X. Qin $(\triangle)$ Department of Clinical Laboratory, First Affiliated Hospital of Guangxi Medical University, 6 Shuangyong Road,

Nanning 530021, Guangxi, People's Republic of China

e-mail: lis8858@126.com

e-mail: qinxue919@126.com

Y. Yan

e-mail: xueying201120521@163.com

H. Liang

e-mail: lianghongjie2004@163.com

R. Li

e-mail: 2609025345@qq.com

L. Xie

e-mail: 83351023@qq.com

M. Li

e-mail: 715085562@qq.com
CC: $\mathrm{OR}=1.00,95 \% \mathrm{CI}=0.91-1.10, P=0.943$; TC vs. TT:

$\mathrm{OR}=0.97,95 \% \mathrm{CI}=0.92-1.04, P=0.396$, Fig. 1 ; TT vs. TC/ $\mathrm{CC}: \mathrm{OR}=1.00,95 \% \mathrm{CI}=0.91-1.12, P=0.874$; TT/TC vs. CC: $\mathrm{OR}=0.98,95 \% \mathrm{CI}=0.94-1.03, P=0.486)$. In the subgroup analysis according to ethnicity, the results suggested that XRCC3 Thr241Met polymorphism was not associated with the risk of ovarian cancer in Caucasians population. No significant association was found between the XRCC3 $\mathrm{Thr} 241$ Met polymorphism and the risk of ovarian cancer. Given the limited sample size and ethnicities included in the metaanalysis, further large scaled and well-designed studies are needed to confirm our results.

Keywords $X R C C 3$ - Polymorphism · Ovarian cancer . Meta-analysis

\section{Introduction}

Ovarian cancer is the eighth most commonly diagnosed cancer and the fifth leading cause of cancer death in the females worldwide with an estimated 225,500 new cases and 140,200 deaths every year $[1,2]$. Though there are many advances in the classification, diagnosis, and treatment of ovarian cancer, it is still difficult to diagnose early and causes serious damage to public health $[3,4]$. Since the low rate of early detection, the late clinical manifestation, and the lack of effective treatments, the 5-year survival rate for ovarian cancer patients is only about $40 \%$ [1]. What is worse, the mechanism of ovarian carcinogenesis is not unclear yet, and multiple factors may increase the risk of developing ovarian cancer, such as age, gravidity, tubal ligation, number of ovulatory cycles, family history of ovarian cancer as well as lifestyle factors [3, 5]. However, not all of those who expose to those risk factors develop ovarian cancer, which suggests genetic factors may also play an important role in the host's susceptibility to 
ovarian cancer [6]. Candidate genes include the insulin receptor substrate 1 [7], lysyl oxidase G473A [8], vitamin D receptor [9], and MTHFR C677T [2].

Another candidate gene that has received a lot of attention is the X-ray repair cross-complementing group 3(XRCC3). $X R C C 3$, located at chromosome $14 \mathrm{q} 32.3$, is a member of DNA repair genes and involved in maintaining the stability of genome by homologous recombination repair for DNA doublestrand breaks $[10,11]$. The Thr241Met is the most common polymorphism of $X R C C 3$, which substitutes at codon 241 in exon 7, with a $\mathrm{C}$ to $\mathrm{T}$ transition (XRCC3-18067C/T, rs861539) $[11,12]$. We called this SNP in the XRCC3 gene "-18067C/T" for short in this meta-analysis. Previous studies were performed to investigate the relationship between the XRCC3-18067C/T polymorphism and ovarian cancer risk [13-16]. However, the results were controversial, which might be caused by the limitation of individual studies. To assess the association between XRCC3-18067C/T polymorphism and ovarian cancer risk more precisely, we conducted a meta-analysis.

\section{Materials and methods}

\section{Search strategy}

To identify all currently available studies on the association between XRCC3-18067C/T polymorphism and ovarian cancer risk, we conducted an extensive search in PubMed, Excerpta Medica Database (Embase), Chinese National Knowledge Infrastructure database, and Chinese Biomedical Literature Database before August 2013 by using the terms as follows: "XRCC3" or "X-ray cross complementing group 3" in combination with "polymorphism," "polymorphisms," "variant," or "mutation" in combination with "ovarian cancer," "ovarian carcinoma," or "ovarian tumor" for all publications. There was no language limitation. Additional studies were identified by a hand search of the references of original studies. Review articles were also examined to find more eligible studies.

\section{Inclusion and exclusion criteria}

Studies to be included in the meta-analysis were required to meet the following criteria: (a) it assessed the association between the XRCC3-18067C/T polymorphism and ovarian cancer, (b) a case-control design, (c) the genotype distribution among the control population was consistent with HardyWeinberg Equilibrium (HWE), and (d) sufficient reported genotypic frequencies in both cases and controls for estimating an odds ratio (OR) with its $95 \%$ confidence interval (95\% $\mathrm{CI})$. The following exclusion criteria were used for excluding studies: (a) control population including patients, (b) studies contained duplicate data, and (c) case reports or reviews.

\section{Data extraction}

Information was carefully extracted from all eligible publications by two authors independently according to the inclusion criteria listed above, disagreements were resolved through consensus, if could not reached agreement, another author was consulted to resolve the debate. The following information was extracted: the name of first author, year of publication, country of origin, ethnicity of the population, genotyping methods, source of the control group, sample size of cases. Different ethnicities were categorized as Caucasians and mixed.

Statistical analysis

The possible association between the XRCC3-18067C/T polymorphism and ovarian cancer susceptibility was measured by the pooled ORs with corresponding $95 \%$ CIs in five genetic models: allele contrast (T vs. C), homozygote (TT vs. $\mathrm{CC}$ ), heterozygote (TC vs. CC), recessive (TT vs. TC/ CC), and dominant (TT/TC vs. CC) models, respectively. Heterogeneity was checked by the Chi-square based Q statistic. If the result of the heterogeneity test $P<0.10$, suggested the between-study heterogeneity was existed, ORs were pooled by random-effects model (DerSimonian and Laird method) [17]. Otherwise, the fixed-effects model (the Mantel-Haenszel method) was used [18]. In addition, the effect of heterogeneity was quantified also by using $I^{2}$ value [19]. If obvious heterogeneity existed $\left(I^{2}\right.$ value $>50 \%$ or $\left.P<0.10\right)$, the overall estimate of risk was calculated by the random-effects model; when obvious heterogeneity was absent, the fixed-effects model was used.

The HWE was assessed by using a professional web-based program (http://ihg2.helmholtz-muenchen.de/cgibin/hw/ hwa1.pl) to confirm whether the genotype distribution of XRCC3-18067C/T in controls agreed with HWE if $P>0.05$ suggests the controls followed HWE balance. Egger's linear regression test was applied to examine the possible publication bias, Egger's test $(P<0.05$ was considered representative of statistically significant publication bias) [20]. Publication bias was also tested by visual observation of funnel plot [21]. Statistical analysis was undertaken using the program STATA Software (version 9.2, Stata Corp). Two-sided $P<0.05$ was considered statistically significant.

\section{Results}

Study characteristics

After carefully selecting based on the search criteria above, a total of four publications including seven studies with 3,635 cases and 5,473 controls were included in our meta-analysis 
Table 1 General characteristics of studies included in the meta-analysis

\begin{tabular}{llllllll}
\hline First author & Year & Country & Ethnicity & Method of genotyping & Source of control & Sample size (case control) & HWE of control \\
\hline Auranen(a) & 2005 & UK & Caucasian & TaqMan & PB & $1139 / 1614$ & 0.395 \\
Auranen(b) & 2005 & USA & Caucasian & TaqMan & PB & $270 / 344$ & 0.111 \\
Auranen(c) & 2005 & Danish & Caucasian & TaqMan & PB & $361 / 891$ & 0.080 \\
Hormazabal & 2012 & Chile & Mix & TaqMan & PB & $87 / 570$ & 0.172 \\
Beesley(a) & 2007 & Australia & Caucasian & PCR-RFLP & PB & $504 / 972$ & 0.326 \\
Beesley(b) & 2007 & Australia & Caucasian & PCR-RFLP & PB & $731 / 747$ & 0.950 \\
Webb & 2005 & Australia & Caucasian & PCR & HB & $543 / 335$ & 0.420 \\
\hline
\end{tabular}

$P C R$-RFLP PCR-restriction fragment length polymorphism, $H W E$ Hardy-Weinberg equilibrium, $H B$ hospital based, $P B$ population based

[13-16], all of these publications were written in English. There were six studies of Caucasians and one mixed. The genotype distribution in the controls of all the studies included in the meta-analysis were consistent with HWE (all $P>0.05$ ). The study characteristics included in the meta-analysis were listed in Table 1.

\section{Quantitative synthesis of data}

The main results of the meta-analysis were listed in Table 2 . Meta-analysis of the total studies showed that there was no association between XRCC3 Thr241Met polymorphism and risk of ovarian cancer under all five genetic models in overall population (T vs. C: $\mathrm{OR}=0.99,95 \% \mathrm{CI}=0.96-1.03$, $P=0.752$; TT vs. $\mathrm{CC}: \mathrm{OR}=1.00,95 \% \mathrm{CI}=0.91-1.10$, $P=0.943$; TC vs. TT: $\mathrm{OR}=0.97,95 \% \mathrm{CI}=0.92-1.04$, $P=0.396$, Fig. 1 ; TT vs. TC/CC: $\mathrm{OR}=1.00,95 \% \mathrm{CI}=0.91-$ $1.12, P=0.874$; TT/TC vs. $\mathrm{CC}: \mathrm{OR}=0.98,95 \% \mathrm{CI}=0.94$
$1.03, P=0.486$ ). In the subgroup analysis according to ethnicity, the results suggested that XRCC3 Thr241Met polymorphism was not associated with the risk of ovarian cancer in Caucasians population (only shown homozygote model in Fig. 2). Detailed data are shown in Table 2.

Heterogeneity analysis and publication bias analysis

There was a significant heterogeneity found under heterozygote (TC vs. CC) and dominant (TT/TC vs. CC) genetic models in overall population. To examine the source of heterogeneity, we assessed the dominant model (TT/TC vs. CC) by ethnicity. However, the results showed there was no obvious heterogeneity in the subgroup analyses of both Caucasians and mix, suggesting that ethnicity was the major source of heterogeneity in our meta-analysis.

Funnel plot and Egger's test were used to assess the publication bias in this meta-analysis. Funnel plot is relatively
Table 2 Results of meta-analysis for XRCC3 Thr241Met polymorphism and ovarian cancer risk

$O R$ odds ratio, $C I$ confidence interval, $F$ fixed-effects model, $R$ random-effects model

\begin{tabular}{|c|c|c|c|c|c|c|c|c|}
\hline \multirow[t]{2}{*}{ Comparison } & \multirow[t]{2}{*}{ Population } & \multirow[t]{2}{*}{$N$} & \multicolumn{3}{|c|}{ Test of association } & \multirow[t]{2}{*}{ Model } & \multicolumn{2}{|c|}{ Test of heterogeneity } \\
\hline & & & OR & $95 \% \mathrm{CI}$ & $P$ value & & $P$ value & $I^{2}$ \\
\hline \multirow[t]{3}{*}{ T vs. C } & Overall & 7 & 0.99 & $0.96-1.03$ & 0.752 & $\mathrm{~F}$ & 0.156 & 35.6 \\
\hline & Mix & 1 & 1.33 & $1.03-1.71$ & 0.027 & $\mathrm{~F}$ & - & - \\
\hline & Caucasians & 6 & 1.00 & $0.96-1.03$ & 0.507 & $\mathrm{~F}$ & 0.534 & 0 \\
\hline \multirow[t]{3}{*}{ TT vs. CC } & Overall & 7 & 1.00 & $0.91-1.10$ & 0.943 & $\mathrm{~F}$ & 0.111 & 42.0 \\
\hline & Mix & 1 & 2.83 & $1.43-4.91$ & 5.62 & $\mathrm{~F}$ & - & - \\
\hline & Caucasians & 6 & 0.98 & $0.89-1.08$ & 0.658 & $\mathrm{~F}$ & 0.933 & 0 \\
\hline \multirow[t]{3}{*}{ TC vs. CC } & Overall & 7 & 0.97 & $0.92-1.04$ & 0.396 & $\mathrm{R}$ & 0.068 & 48.8 \\
\hline & Mix & 1 & 1.08 & $0.81-1.44$ & 0.590 & $\mathrm{~F}$ & - & - \\
\hline & Caucasians & 6 & 0.97 & $0.91-1.03$ & 0.336 & $\mathrm{R}$ & 0.045 & 55.9 \\
\hline \multirow[t]{3}{*}{ TT vs. TC/CC } & Overall & 7 & 1.00 & $0.91-1.12$ & 0.874 & $\mathrm{~F}$ & 0.131 & 39.1 \\
\hline & Mix & 1 & 2.83 & $1.40-5.75$ & 0.004 & $\mathrm{~F}$ & - & - \\
\hline & Caucasians & 6 & 0.99 & $0.89-1.10$ & 0.852 & $\mathrm{~F}$ & 0.908 & 0 \\
\hline \multirow[t]{3}{*}{ TT/TC vs. CC } & Overall & 7 & 0.98 & $0.94-1.03$ & 0.486 & $\mathrm{R}$ & 0.083 & 46.3 \\
\hline & Mix & 1 & 0.99 & $0.96-1.02$ & 0.175 & $\mathrm{~F}$ & - & - \\
\hline & Caucasians & 6 & 0.99 & $0.95-1.02$ & 0.426 & $\mathrm{~F}$ & 0.104 & 45.2 \\
\hline
\end{tabular}




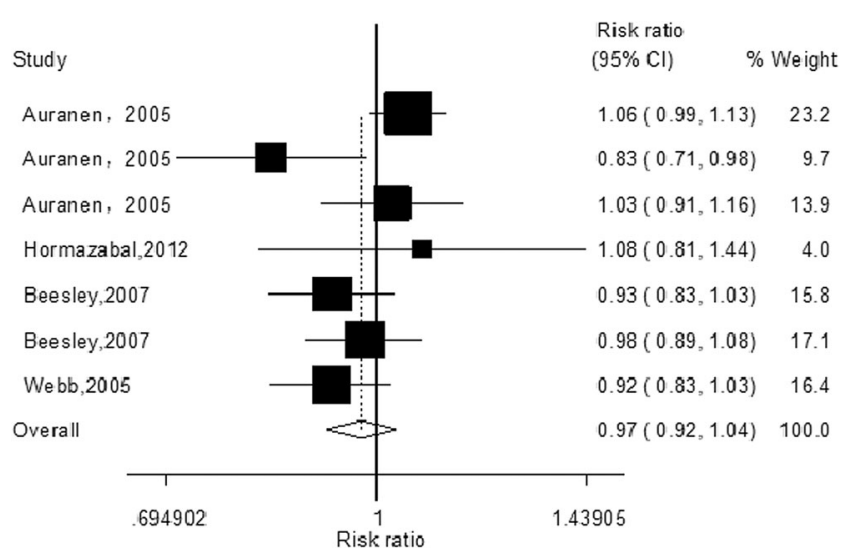

Fig. 1 The forest plot describing the meta-analysis under heterozygous model for the association between XRCC3 $\mathrm{Thr} 241$ Met polymorphism and the risk of ovarian cancer in overall population (TC vs. CC)

straightforward to observe whether the presence of publication bias and Egger's test was provided statistical evidence of symmetries of the plots. As a result, both the shape of the funnel plot and Egger's test value did not suggest any evidence of obvious asymmetry (Fig. 3, all $P>0.05$, data not shown).

\section{Discussion}

It is well known that SNPs are the most common sources of human genetic variation, and they may contribute to an individual's susceptibility to cancer. $X R C C 3$ plays a critical role in maintaining genomic integrity by repairing radiation-induced DNA double-strand breaks, and the XRCC3 polymorphism may affect the DNA repair capacity of its encoded protein, thus, contributes to the development of cancers $[11,12]$.

Since the identification of XRCC3 Thr241Met polymorphism, a growing number of studies suggested that XRCC3 Thr241Met polymorphism plays an important role in the development of cancers, such as glioma [22], hepatocellular

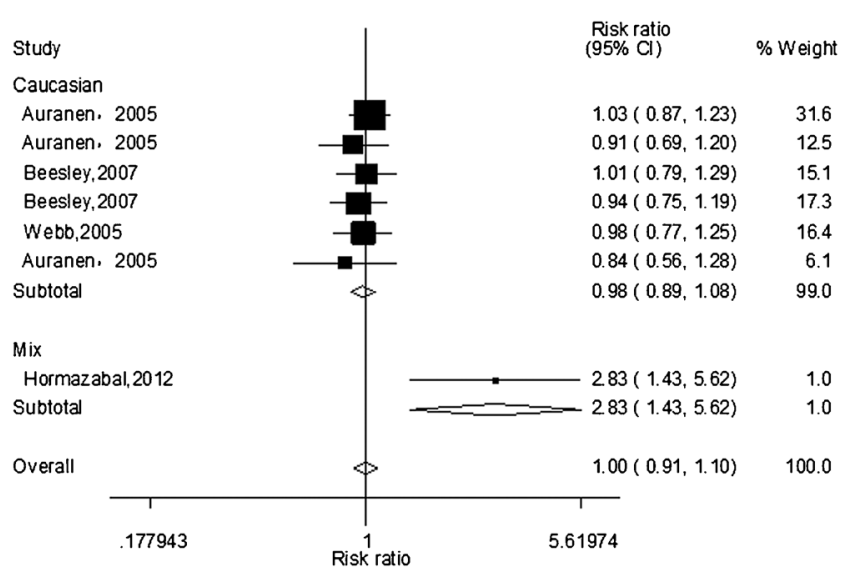

Fig. 2 The forest plot describing the meta-analysis under homozygous model for the association between XRCC3 Thr241 Met polymorphism and the risk of ovarian cancer in Caucasians (TT vs. CC)

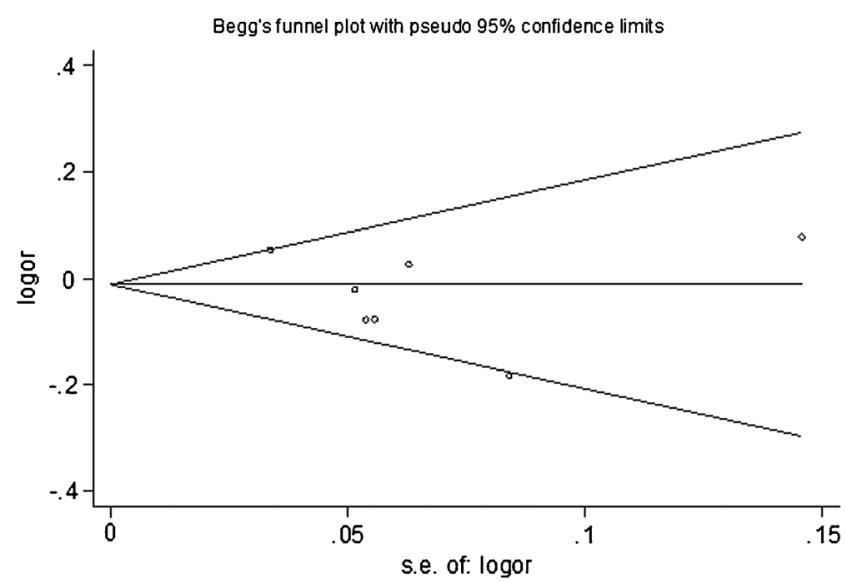

Fig. 3 Begg funnel plot for publication bias test for the association between XRCC3 Thr241 Met polymorphism and the risk of ovarian cancer under heterozygous model (TC vs. CC). Each point represents a separate study for the indicated association. $\log [O R]$ natural logarithm of OR. Horizontal line means effect size

carcinoma [23], head and neck cancer [24], lung cancer [25], and so on. Many published studies that aim at the role of XRCC3 Thr241Met polymorphism in ovarian cancer risk have been performed, but the results are controversial. Different study designs, various methodologies, and different population backgrounds would inevitably increase the risk, which should be responsible for the controversial conclusions. However, meta-analysis is a powerful tool for analyzing cumulative data of studies which the individual sample sizes are small and the statistical power is low. No meta-analysis evaluating on the association between the XRCC3 Thr241Met polymorphisms and ovarian cancer risk has been performed. So, we carried out a meta-analysis.

A total of four publications including seven studies with 3 , 635 cases and 5,473 controls were included in our metaanalysis [13-16]. The results of the meta-analysis showed that XRCC3 Thr241Met polymorphism was not associated with ovarian cancer risk in the overall populations. Subgroup analysis based on ethnicity indicated that XRCC3 Thr241Met polymorphism was not a risk factor for Caucasians, but is a risk for mix. The results about mix may not be accurate because only one study was found about mix; the sample size is too small to explain the conclusion.

The heterogeneity is a very important part of a metaanalysis, and finding the possible sources for the high heterogeneity is very important [26,27]. The studies included in our meta-analysis probably have different genetic backgrounds, environmental exposures, methodology, and sample size, thus, caused the inconsistent conclusions. In this study, obvious heterogeneity between-study was found under heterozygote and dominant genetic models in the overall population. To explore the possible sources for the heterogeneity in the meta-analysis, we performed subgroup analysis by ethnicity. Through subgroup analysis, we found that ethnicity was the major source of the heterogeneity in our meta-analysis, which 
could be explained by the race-specific effect of $X R C C 3$ Thr241Met polymorphism and the susceptibility of ovarian cancer that is because different countries may have different genetic backgrounds and environmental exposures. Publication bias is another important factor which may have a negative effect on the meta-analysis. Both Funnel plot and Egger's test were used to assess the publication bias of our metaanalysis. The shape of funnel plot and statistical results show no obvious publication bias found; this indicates that the publication bias has little effect on the results of our study, and the results of our meta-analysis are relatively stable.

There are several limitations in our meta-analysis that should be considered. Firstly, the sample sizes are relatively small; there are only seven studies with a total of 3,635 cases and 5,473 controls included in our meta-analysis. More studies with large sample size and well-designed are needed to further identify this association more comprehensively. Secondly, studies included in the present meta-analysis mainly provided data towards Caucasians in the light of the race-specific association probably exist; other ethnicities including Asians, Africans, and others should be investigated in future studies. Thirdly, subgroup analyses according to age, radiation exposure, histological types, and other elements have not been performed due to insufficient relevant data available in the primary studies. Finally, only published and English studies were included in this study; thus, publication and potential language biases may occur.

In summary, our meta-analysis systematically analyzed the association between XRCC3 Thr241Met polymorphism and the risk of ovarian cancer. No significant association was found between the XRCC3 Thr241 Met polymorphism and the risk of ovarian cancer. Given the limited sample size and ethnicities included in the meta-analysis, further larger scaled and well-designed studies are needed to confirm our results.

\section{Conflicts of interest None}

Open Access This article is distributed under the terms of the Creative Commons Attribution License which permits any use, distribution, and reproduction in any medium, provided the original author(s) and the source are credited.

\section{References}

1. Jemal A, Bray F, Center MM, Ferlay J, Ward E, Forman D. Global cancer statistics. CA Cancer J Clin. 2011;61:69-90.

2. Ma C, Liu Y, Zhang W, Liu P. The association between MTHFR c677t polymorphism and ovarian cancer risk: a meta-analysis of 18 , 628 individuals. Mol Biol Rep. 2013;40:2061-8.

3. Hennessy BT, Coleman RL, Markman M. Ovarian cancer. Lancet. 2009;374:1371-82.

4. Bertone-Johnson ER. Epidemiology of ovarian cancer: a status report. Lancet. 2005;365:101-2.

5. Brekelmans CT. Risk factors and risk reduction of breast and ovarian cancer. Curr Opin Obstet Gynecol. 2003;15:63-8.
6. Pennington KP, Swisher EM. Hereditary ovarian cancer: beyond the usual suspects. Gynecol Oncol. 2012;124:347-53.

7. Ding YC, McGuffog L, Healey S, Friedman E, Laitman Y, PaluchShimon S, et al. A nonsynonymous polymorphism in IRS1 modifies risk of developing breast and ovarian cancers in BRCA1 and ovarian cancer in BRCA2 mutation carriers. Cancer Epidemiol Biomarkers Prev. 2012;21:1362-70.

8. Wu J, Cai C, Tong D, Hou H. Lysyl oxidase g473a polymorphism is associated with increased risk of ovarian cancer. Genet Test Mol Biomarkers. 2012;16:915-9.

9. Liu Y, Li C, Chen P, Li X, Li M, Guo H, et al. Polymorphisms in the vitamin D receptor (VDR) and the risk of ovarian cancer: a metaanalysis. PLoS One. 2013;8:e66716.

10. Thompson LH, Schild D. Recombinational DNA repair and human disease. Mutat Res. 2002;509:49-78.

11. Griffin CS, Simpson PJ, Wilson CR, Thacker J. Mammalian recombination-repair genes $\mathrm{xrcc} 2$ and $\mathrm{xrcc} 3$ promote correct chromosome segregation. Nat Cell Biol. 2000;2:757-61.

12. Matullo G, Guarrera S, Carturan S, Peluso M, Malaveille C, Davico $\mathrm{L}$, et al. DNA repair gene polymorphisms, bulky DNA adducts in white blood cells and bladder cancer in a case-control study. Int $\mathrm{J}$ Cancer. 2001;92:562-7.

13. Auranen A, Song H, Waterfall C, Dicioccio RA, Kuschel B, Kjaer SK, et al. Polymorphisms in DNA repair genes and epithelial ovarian cancer risk. Int J Cancer. 2005; 117:611-8.

14. Gonzalez-Hormazabal P, Reyes JM, Blanco R, Bravo T, Carrera I, Peralta $\mathrm{O}$, et al. The bard1 cys557ser variant and risk of familial breast cancer in a south-American population. Mol Biol Rep. 2012;39:8091-8.

15. Beesley J, Jordan SJ, Spurdle AB, Song H, Ramus SJ, Kjaer SK, et al. Association between single-nucleotide polymorphisms in hormone metabolism and DNA repair genes and epithelial ovarian cancer: results from two Australian studies and an additional validation set. Cancer Epidemiol Biomarkers Prev. 2007;16:2557-65.

16. Webb PM, Hopper JL, Newman B, Chen X, Kelemen L, Giles GG, et al. Double-strand break repair gene polymorphisms and risk of breast or ovarian cancer. Cancer Epidemiol Biomarkers Prev. 2005; 14:319-23.

17. DerSimonian R, Laird N. Meta-analysis in clinical trials. Control Clin Trials. 1986;7:177-88.

18. Mantel N, Haenszel W. Statistical aspects of the analysis of data from retrospective studies of disease. J Natl Cancer Inst. 1959;22:719-48.

19. Higgins JP, Thompson SG. Quantifying heterogeneity in a metaanalysis. Stat Med. 2002;21:1539-58.

20. Egger M, Davey Smith G, Schneider M, Minder C. Bias in metaanalysis detected by a simple, graphical test. BMJ. 1997;315:629-34.

21. Begg CB, Mazumdar M. Operating characteristics of a rank correlation test for publication bias. Biometrics. 1994;50:1088-101.

22. Lin J, Kou Y. Association between the thr241met polymorphism of $\mathrm{x}$-ray repair cross-complementing group 3 gene and glioma risk: evidence from a meta-analysis based on 4,136 cases and 5,233 controls. Tumour Biol 2013.

23. Wu D, Jiang H, Yu H, Xu D, Liang J, Jin J. Significant association between xrcc3 $\mathrm{c} 241 \mathrm{t}$ polymorphism and increased risk of hepatocellular carcinoma: a meta-analysis. Tumour Biol 2013.

24. Yin QH, Liu C, Li L, Zu XY, Wang YJ. Association between the xrcc $3 \mathrm{t} 241 \mathrm{~m}$ polymorphism and head and neck cancer susceptibility: a meta-analysis of case-control studies. Asian Pac J Cancer Prev. 2012;13:5201-5.

25. Xu YH, Gu LP, Sun YJ, Cheng BJ, Lu S. No significant association between the XRCC3 Thr241met polymorphism and lung cancer risk: a meta-analysis. Tumour Biol. 2013;34:865-74.

26. Zintzaras E, Lau J. Synthesis of genetic association studies for pertinent gene-disease associations requires appropriate methodological and statistical approaches. J Clin Epidemiol. 2008;61:634-45.

27. Ioannidis JP, Patsopoulos NA, Evangelou E. Uncertainty in heterogeneity estimates in meta-analyses. BMJ. 2007;335:914-6. 